\title{
Morphodynamic Analysis of Cerebral Aneurysm Pulsation From Time-Resolved Rotational Angiography
}

\author{
Chong Zhang, Maria-Cruz Villa-Uriol, Member, IEEE, Mathieu De Craene, Jose M. Pozo, and \\ Alejandro F. Frangi*, Senior Member, IEEE
}

\begin{abstract}
This paper presents a technique to estimate and model patient-specific pulsatility of cerebral aneurysms over one cardiac cycle, using 3D rotational $X$-ray angiography (3DRA) acquisitions. Aneurysm pulsation is modeled as a time varying $B$-spline tensor field representing the deformation applied to a reference volume image, thus producing the instantaneous morphology at each time point in the cardiac cycle. The estimated deformation is obtained by matching multiple simulated projections of the deforming volume to their corresponding original projections. A weighting scheme is introduced to account for the relevance of each original projection for the selected time point. The wide coverage of the projections, together with the weighting scheme, ensures motion consistency in all directions. The technique has been tested on digital and physical phantoms that are realistic and clinically relevant in terms of geometry, pulsation and imaging conditions. Results from digital phantom experiments demonstrate that the proposed technique is able to recover subvoxel pulsation with an error lower than $10 \%$ of the maximum pulsation in most cases. The experiments with the physical phantom allowed demonstrating the feasibility of pulsation estimation as well as identifying different pulsation regions under clinical conditions.
\end{abstract}

Index Terms-Image registration, motion analysis, three-dimensional rotational angiography, X-ray imaging.

Manuscript received November 22, 2008; revised December 24, 2008. First published January 19, 2009; current version published June 24, 2009. This work was supported in part by Philips Healthcare, Best, The Netherlands, in part by the CENIT-CDTEAM grant funded by the Spanish Ministry Of Science and Innovation (MICINN-CDTI), and partially generated in the framework of the @ neurIST Integrated Project, which is co-financed by the European Commission (IST-027703). Asterisk indicates corresponding author.

C. Zhang, M. C. Villa-Uriol, and J. M. Pozo are with the Center for Computational Imaging and Simulation Technologies in Biomedicine (CISTIB), Universitat Pompeu Fabra, 08003 Barcelona, Spain, and also with the Networking Research Center on Bioengineering, Biomaterials, and Nanomedicine (CIBERBBN), 08003 Barcelona, Spain (e-mail: chong.zhang@upf.edu; cruz.villa@upf. edu; jose.pozo@upf.edu).

M. De Craene is with the Networking Research Center on Bioengineering, Biomaterials and Nanomedicine (CIBER-BBN), 08018 Barcelona, Spain, and also with the Center for Computational Imaging and Simulation Technologies in Biomedicine (CISTIB), Information and Communications Technologies Department, Universitat Pompeu Fabra, 08018 Barcelona, Spain (e-mail: mathieu. decraene@upf.edu).

*A. F. Frangi is with the Center for Computational Imaging and Simulation Technologies in Biomedicine (CISTIB), Universitat Pompeu Fabra, 08018 Barcelona, Spain, and also with the Networking Research Center on Bioengineering, Biomaterials, and Nanomedicine (CIBER-BBN), 08018 Barcelona, Spain (e-mail: alejandro.frangi@upf.edu).

Color versions of one or more of the figures in this paper are available online at http://ieeexplore.ieee.org.

Digital Object Identifier 10.1109/TMI.2009.2012405

\section{INTRODUCTION}

C EREBRAL aneurysms are pathological enlargements of brain arteries commonly located at the Circle of Willis [1]. When they rupture, spontaneous subarachnoid hemorrhage usually follows, causing high morbidity and mortality rates [1], [2]. Morphological characterization has been reported to provide indicators for monitoring the growth of intracranial aneurysms, as well as for correlating it with rupture events [3]-[5]. However, such techniques only consider static morphological information such as size and aspect ratio. On the other hand, it has been observed that aneurysms pulsate over the cardiac cycle [6]-[8] and rupture sites coincide with the areas of pulsation [9]-[11]. If such correlation exists, the availability of a robust morphodynamic analysis tool has the potential of impacting treatment selection and preoperative planning of cerebral aneurysms. Nonetheless, a small motion range that can go below the image resolution makes the morphodynamic analysis practically challenging. Dempere et al. [12] proposed a motion estimation method from dynamic biplane digital subtraction angiography (DSA) using 2D nonrigid image registration. Their recent work [13] extended this approach by postprocessing the recovered motion curve in the Fourier domain. Since DSA images are captured from a single point of view, motion was only partially estimated. Various techniques have been proposed for motion estimation and dynamic reconstruction. Most of them, e.g., dynamic CT [14], use a subset of projections belonging to the same motion state according to a time reference signal such as the electrocardiogram (ECG), and use this subset to reconstruct the object at that motion state. However, they are not tailored to wall motion estimation in cerebral aneurysms.

Movassaghi et al. [15] presented in-human 4D coronary artery reconstructions using an ECG-gated 3D tomographic reconstruction from projection images [16]. The promising results counterbalance the strong requirements in the imaging protocol and preprocessing. Schäfer et al. [17] reconstructed moving coronary arteries by shifting voxel positions according to a motion vector field. This method is only applicable to voxel driven cone-beam filtered back-projection (FBP) reconstruction approaches [18], [19] with an a priori known motion model. To perform gated reconstruction, the authors introduced a weighting factor into the FBP formulation according to the cardiac phase of the projections. Unfortunately, streak artifacts associated to FBP algorithms were not completely eliminated. 
Blondel et al. [20], [21] computed a 4D B-spline deformation field of coronary arteries. A static 3D centerline model reconstructed at one cardiac phase was used to fit all projection images. Nonetheless, the centerline model is not suitable for aneurysm geometries in providing local deformation. Zeng $e t$ al. [22] also estimated 4D thorax respiratory motion during one cone-beam computed tomography (CBCT) acquisition using $B$-spline deformation. The need for two acquisitions (the reference CT and the CBCT projections) does not only increase patient exposure to radiation, but also might lead to misinterpret intrinsic organ motion as respiratory motion.

Several approaches were proposed for performing 2D motion correction and then reconstruct the 3D image from the motioncompensated 2D projections [23], [24]. They have been successfully applied to coronary artery or stent reconstruction. However, we aim at recovering the $3 \mathrm{D}$ motion instead of removing it from the $2 \mathrm{D}$ projections. Furthermore, neither constraining motion to be affine [24] nor using the centerline model [23] are suitable options for the analysis of aneurysms. We therefore propose to model the aneurysm pulsation in the $3 \mathrm{D}$ space rather than in the space of the individual 2D projections.

Our previous work [25] presented a general framework to estimate 3D aneurysmal wall motion over one cardiac cycle from 3D rotational X-ray angiography (3DRA) acquisitions. In this paper, the presented methodology models cerebral aneurysm pulsation as a time varying $B$-spline tensor field. It is applied to a reference volumetric image to estimate the instantaneous deformation at any time point in the cardiac cycle. The optimal $B$-spline transformation is obtained by matching multiple simulated projections of the deforming volume to the corresponding original projections. The transformation continuity and smoothness not only preserves the image resolution of the 3DRA reference volume, but also enables to recover subvoxel pulsation. A weighting scheme is introduced to account for the relevance of each projection to the estimated time point. The wide coverage of the projections, together with the weighting scheme, ensures motion consistency in all directions. The use of only one standard acquisition performed during an endovascular treatment does not expose patient to additional radiation risk and facilitates its clinical take-up.

The subsequent sections are organized as follows. In Section II, the proposed methodology is explained in detail. Section III shows the experiments carried out on various digital phantoms for the quantitative evaluation of our method. Section IV reports the results obtained from physical phantoms, to demonstrate the feasibility of our method. Discussion and conclusions are summarized in Sections V and VI, respectively.

\section{METHOD}

During a 3DRA acquisition, a rotational sequence of cone beam X-ray projections are first obtained and then used to reconstruct a $3 \mathrm{D}$ volumetric image. In addition, a physiological signal (e.g., ECG or blood pressure) synchronized to the projections can be recorded. Typically, more than 100 projections are obtained during $4 \mathrm{~s}$, at constant time intervals and uniformly distributed over more than $200^{\circ}$ along a circular trajectory. Thus, the vascular motion occurring during the acquisition is captured

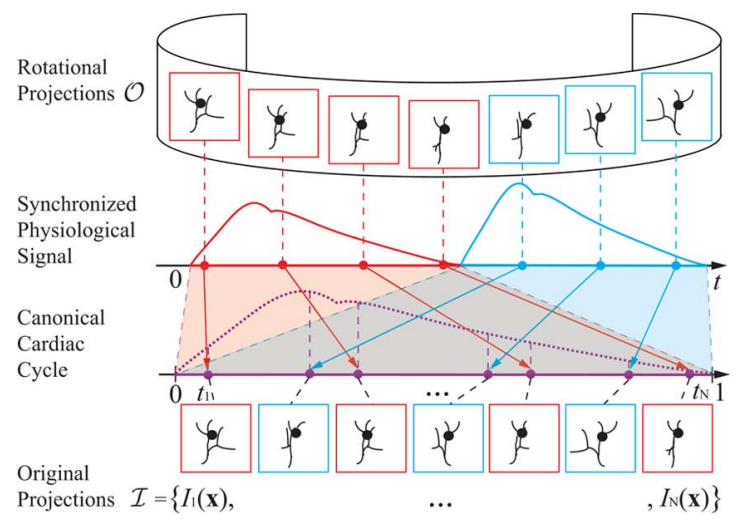

Fig. 1. Creation of a canonical cardiac cycle according to the original rotational projections and the synchronized physiological signal.

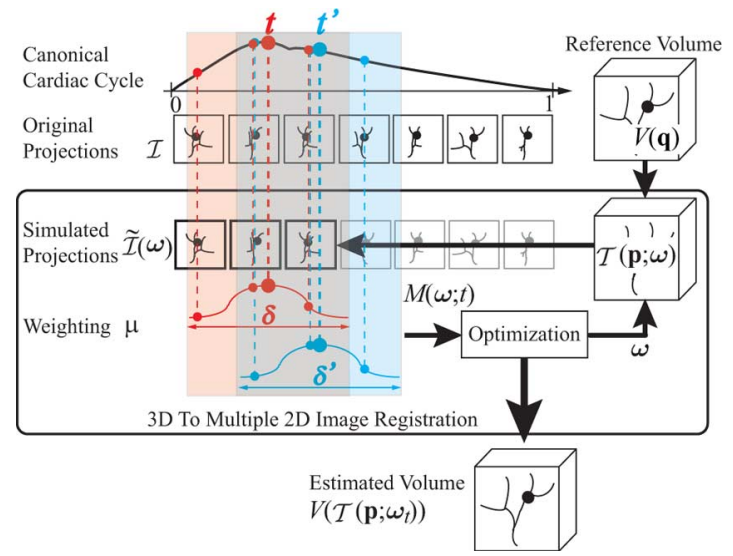

Fig. 2. 3D-to-multiple-2D image registration procedure for the canonical cardiac cycle. Two weighting windows are shown to illustrate the weighting scheme at two estimated time points, $t$ and $t^{\prime}$.

in the $2 \mathrm{D}$ projections. However, since only a single volume is reconstructed, it does not provide the 3D dynamic morphology.

The objective of our work is to retrieve the time-resolved morphology of the imaged region by combining the 3D volume, the projections and a synchronized physiological signal. First, according to the cardiac cycles in the physiological signal, the projections are reordered to build one normalized cardiac cycle containing all of them (original projections). This step is detailed in Section II-A (Fig. 1). Then, at each time point in the normalized cardiac cycle, a 3D-to-multiple-2D image registration procedure is performed (Fig. 2). During this step, the $3 \mathrm{D}$ volume (reference volume) is iteratively deformed (Section II-B) to maximize the similarity (Section II-D) between the original projections and their corresponding simulated projections. The simulated projections are calculated from the deformed volume through a ray casting process (Section II-C). The contribution to the similarity from each pair of original and simulated projections is weighted according to the temporal difference between the original projection and the current estimation.

\section{A. Creation of a Canonical Cardiac Cycle}

Initially, the original projection sequence $\mathcal{O}$ of size $N$ is used to reconstruct the reference volume. As shown in Fig. 1, a physiological signal is recorded synchronously with $\mathcal{O}$. Since in prac- 
tice, the waveform of the physiological signal can vary from cycle to cycle, each cycle of this signal is extracted and its period is then linearly normalized to a time interval $[0,1)$. All images in $\mathcal{O}$ are sorted by their normalized timestamps to build one canonical cardiac cycle as

$$
\mathcal{I}=\left\{I_{k}(\mathbf{x}) \mid k=1 \ldots N, \mathbf{x} \in \mathcal{S}_{k} \subset \mathbb{R}^{3}\right\}
$$

where the reordered image $I_{k}(\mathbf{x})$ lies on the corresponding projection plane $\mathcal{S}_{k}$, and is located within the canonical cardiac cycle at time $t_{k}$

$$
0 \leq t_{1} \leq \cdots \leq t_{k} \leq \cdots \leq t_{N}<1
$$

\section{B. Deformation Model}

The motion at a given instant of time $t$ is represented as a deformed reference volume

$$
V_{\mathrm{d}}(\mathbf{p} ; t)=V\left(\mathcal{T}\left(\mathbf{p} ; \omega_{t}\right)\right)
$$

where the transformation $\mathcal{T}: \mathbb{R}^{3} \mapsto \mathbb{R}^{3}$ maps a point $\mathbf{p}$ in $V_{\mathrm{d}}(\mathbf{p})$ to the point $\mathbf{q}$ in $V(\mathbf{q}), \boldsymbol{\omega}_{t}$ denotes the time-varying transformation parameters at $t$. We have chosen a $B$-spline based transformation [26], [27], because it is not only smooth and continuous but also computationally efficient due to the local control of the basis functions. The transformation for any set of parameters $\boldsymbol{\omega}$ is given by

$$
\mathcal{T}(\mathbf{p} ; \boldsymbol{\omega})=\mathbf{p}+\sum_{\mathbf{c}} B\left(\frac{\mathbf{p}-\mathbf{p}^{\mathbf{c}}}{\boldsymbol{\Delta}^{\mathbf{c}}}\right) \boldsymbol{\omega}^{\mathbf{c}}
$$

where $B(\cdot)$ is the $3 \mathrm{D}$ tensor product of $1 \mathrm{D}$ cubic $B$-spline basis functions, defined on a sparse grid of control points $\mathbf{p}^{\mathbf{c}}$, being $\mathbf{c}$ the index of the control points, and $\Delta^{\mathbf{c}}$ the width of the functions. Because of these short basis functions with limited support, the $B$-spline transformation can only represent a subset of all possible deformation fields [26]. However, this limitation of the basis functions also provides an implicit regularization of the transformation.

\section{Generation of Simulated Projections}

In this paper, a simulated projection $\tilde{I}(\mathbf{x})$ is calculated from $V(\mathbf{p})$ through a ray casting procedure [28], [29] in order to approximate the $\mathrm{X}$-ray attenuation imaging process [30]. Starting from each pixel $\mathbf{x}$ in the projection, a ray $\mathcal{L}_{\mathbf{s}, \mathbf{x}}$ is casted through the volume to meet the X-ray source point $\mathbf{s}$ (Fig. 3). The pixel intensity of $\tilde{I}(\mathbf{x})$ is then defined as the integral of the volume intensities $V(\boldsymbol{\gamma})$ for every point $\boldsymbol{\gamma}$ lying on $\mathcal{L}_{\mathbf{s}, \mathbf{x}}$

$$
\tilde{I}(\mathbf{x})=\int_{\mathcal{L}_{\mathbf{s}, \mathbf{x}}} V(\boldsymbol{\gamma}) d \boldsymbol{\gamma} \approx \sum_{\ell} \alpha_{\ell} V\left(\mathbf{p}_{\ell}\right)
$$

where $\ell$ is the index of the sampled voxel points $\mathbf{p}_{\ell}$ along $\mathcal{L}_{\mathbf{s}, \mathbf{x}}$, and $\alpha_{\ell}$ the sampling weight, the distance between two consecutively sampled points $\mathbf{p}_{\ell}$ and $\mathbf{p}_{\ell+1}$. On each of the original projection planes $\mathcal{S}_{k}$, such a simulated projection can be generated by ray casting $V_{\mathrm{d}}(\mathbf{p} ; t)$. The sequence of simulated projections is denoted as

$$
\tilde{\mathcal{I}}=\left\{\tilde{I}_{k}(\mathbf{x}) \mid k=1 \ldots N, \mathbf{x} \in \mathcal{S}_{k} \subset \mathbb{R}^{3}\right\} .
$$

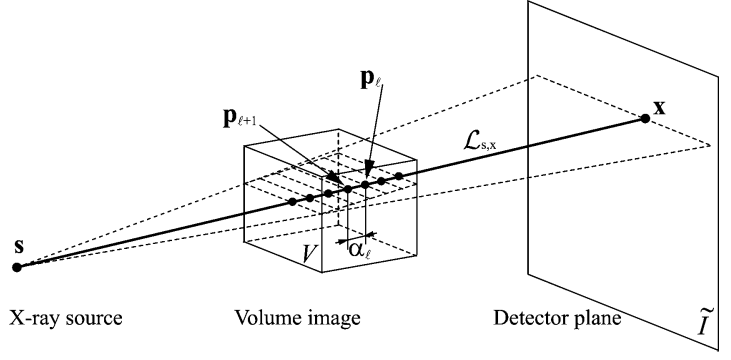

Fig. 3. Overview of the ray casting algorithm to compute the simulated projection $\tilde{I}(\mathbf{x})$.

$\tilde{\mathcal{I}}$ reflects the morphology at time $t$ from various projection views. Note that for each image $\tilde{I}_{k}(\mathbf{x})$, a valid ray region $\mathcal{R}_{k}$ is defined so that only those rays intersecting the volume are evaluated. For the rest of the pixels, their intensity value is set to zero.

\section{Similarity Measures}

During the registration process at an instant of time $t$ in the canonical cardiac cycle, the corresponding deformation parameters $\omega_{t}$ are determined by matching the simulated projections to the original projections

$$
\boldsymbol{\omega}_{t}=\arg \min _{\boldsymbol{\omega}}\left\{M(\boldsymbol{\omega} ; t)=\sum_{k} \mu\left(t, t_{k}\right) D\left(\tilde{I}_{k}(\boldsymbol{\omega}), I_{k}\right)\right\}
$$

where $M(\cdot)$ is the metric that sums up a weighted similarity measure between each original and simulated projection pair, $D(\cdot)$ the similarity metric, and $\mu(\cdot)$ the weighting factor accounting for the temporal proximity between two projections.

1) Similarity Measures Between Two Images: As simulated projections are approximations of the original projections, the image intensity ranges are different, therefore mutual information has been used in our work

$$
D\left(\tilde{I}_{k}(\boldsymbol{\omega}), I_{k}\right)=-\sum_{u} \sum_{v} p(u, v) \log \frac{p(u, v)}{p(u) p(v)}
$$

where $p(u)$ and $p(v)$ are, respectively, the marginal probability distributions of the intensity values of $I_{k}$ and $\tilde{I}_{k}$, and $p(u, v)$ the joint probability distributions of these two images. Here $u, 0<u<H$ and $v, 0<v<\tilde{H}$ are the indices of uniformly sized histogram bins along the respective dimensions of the joint histogram.

Histograms are approximated using Parzen windows for the probability calculation [31], [32]. Let $\beta^{(0)}$ and $\beta^{(3)}$ be a zeroorder and a cubic $B$-spline Parzen windows. The joint histogram is then given by

$$
\begin{aligned}
& p(u, v)=\hat{\alpha} \sum_{\mathbf{x} \in \mathcal{R}_{k}} \beta^{(0)}\left(u-\frac{I_{k}(\mathbf{x})-i_{k}^{\circ}}{\Delta h}\right) \\
& \cdot \beta^{(3)}\left(v-\frac{\tilde{I}_{k}(\mathbf{x} ; \boldsymbol{\omega})-\tilde{i}_{k}^{\circ}}{\Delta \tilde{h}}\right)
\end{aligned}
$$


where $\hat{\alpha}$ is a normalization factor that ensures $\sum p(u, v)=1, i_{k}^{\circ}$ and $\tilde{i}_{k}^{\circ}$ the minimum intensity values, and $\Delta h$ and $\Delta \tilde{h}$ the intensity bin sizes. The marginal histogram of $\tilde{I}_{k}$ is computed as

$$
p(v)=\sum_{u} p(u, v) .
$$

As $I_{k}$ is independent from the transformation, its marginal probability can be precomputed as

$$
p(u)=\hat{\alpha} \sum_{\mathbf{x} \in \mathcal{R}_{k}} \beta^{(0)}\left(u-\frac{I_{k}(\mathbf{x})-i_{k}^{\circ}}{\Delta h}\right) .
$$

2) Weighting Temporal Proximity: For the $k$ th projection plane $\mathcal{S}_{k}, I_{k}$ is acquired at $t_{k}$, whereas $\tilde{I}_{k}(\omega)$ represents the estimated motion state at $t$. If $t_{k}$ is sufficiently close to $t$, the differences between the motion state at these two time points should be small, implying the two images should be very similar. Otherwise, large differences should be expected. Hence, $I_{k}$ should have a reduced impact on determining $\omega$. Thus, the weighting factor $\mu\left(t, t_{k}\right)$ is a symmetric operator centered at $t$ and should satisfy

$$
\left|t-t_{1}\right|>\left|t-t_{2}\right| \Rightarrow \mu\left(t, t_{1}\right)<\mu\left(t, t_{2}\right) \text {. }
$$

In this paper, a cosine power function is used [33]

$$
\mu\left(t, t_{k}\right)= \begin{cases}\cos ^{\lambda}\left(\pi \frac{t-t_{k}}{\delta}\right), & \text { if }\left|t_{k}-t\right|<\frac{\delta}{2} \\ 0, & \text { otherwise. }\end{cases}
$$

This function can be shaped easily by the modification of the exponent. With $0<\lambda \leq 2$, very small values at the window boundaries are avoided. For our experiments, $\lambda=2$ was used. In addition, the finite support of this function window width $\delta$ reduces the computation only to the projections within the time interval $[t-\delta / 2, t+\delta / 2]$.

By combining information from the projections at adjacent time points, motion can be estimated at any time point within the cardiac cycle. Note that it also limits the level of detail of the temporal evolution of the estimated motion due to the smoothing effect introduced by interpolating the projections. Therefore, the value of $\delta$ should be chosen as a trade-off between the local pulsation variation and the number of projections available within this time interval. However, as pulsation is not known a priori, a fixed window width can be chosen for all the selected time points over the cardiac cycle. As shown by our experiments, the presented weighting scheme enforces continuity of the recovered deformation in time, and adequately accommodates for heart rate variations.

\section{E. Optimization Strategy}

The success of image registration depends on the optimization of the image matching metric. A wide range of optimization methods can be considered to minimize the metric in (3). Since the number of parameters characterizing the transformation is large, and the metric is explicitly differentiable, gradient-based methods are a natural choice. The L-BFGS-B method [34] is used, which searches the optimum according to the gradient and a low-rank approximation of the Hessian of the metric. The analytical computation of the metric gradient is detailed in the Appendix.
TABLE I

DESIGN PARAMETERS FOR DigITAL PHANTOM STUDIES

\begin{tabular}{lcc}
\hline Parameters & Type I & Type II \\
\hline Dome shape & Irregular & Spherical \\
Dome diameter $(\mathrm{mm})$ & $8-12$ & 10 \\
Vessel shape & Toroidal & Cylindrical \\
Vessel diameter $(\mathrm{mm})$ & 4 & 4 \\
& & \\
Pulsation waveform & Sinusoidal & Physiological \\
Pulsation frequency $(\mathrm{bpm})$ & $\sim 90$ & $\sim 60$ \\
Pulsation amplitude range $(\mathrm{mm})$ & $0.08-0.48$ & $0.41-0.69$ \\
& & 30 \\
Frame rate $(\mathrm{Hz})$ & 25 & $0.16^{2}$ or $0.31^{2}$ \\
Projection spacing $\left(\mathrm{mm}^{2}\right)$ & $0.16^{2}$ or $0.31^{2}$ & $0.3^{3}$ \\
Volume spacing $\left(\mathrm{mm}^{3}\right)$ & $0.3^{3}$ &
\end{tabular}

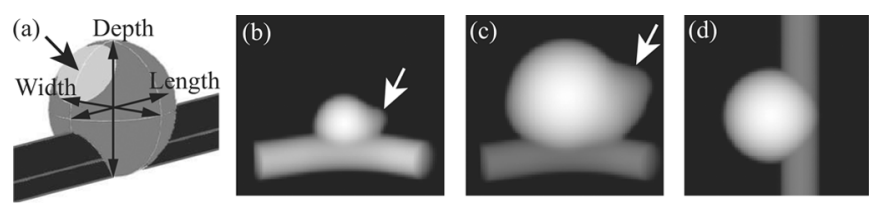

Fig. 4. (a) An illustration of measures referred to as depth, width, and length. Simulated projections of (b), (c) Type I and (d) Type II phantoms. Arrows indicate bleb regions.

\section{DigitAl PHANTOM EXPERIMENTS}

Before analyzing patient data, our method has been validated using digitally simulated aneurysm phantom models, allowing to study the influence of various factors on its performance. Geometry, pulsation, and imaging conditions were simulated to be realistic and clinically relevant. The modeling parameters are summarized in Table I.

\section{A. Experimental Design}

1) Geometry: Two types of phantoms were created as smooth combinations of primitive geometries such as spheres, cylinders, and tori of different sizes (Fig. 4). Type I phantoms presented an emerging bleb on an irregularly shaped dome attached to a toroidal vessel of $4 \mathrm{~mm}$ in diameter, whereas Type II phantoms consisted of a 10-mm-diameter spherical dome attached to a 4-mm-diameter cylindrical vessel. For each phantom, a sequence of geometries was generated to represent the groundtruth pulsation in several cardiac cycles. The phantom geometry smoothly changed according to a predefined pulsation waveform and was sampled at a finite number of time points.

2) Pulsation: For Type I phantoms, periodic sinusoidal waveforms were used to create wall displacements, e.g., Fig. 5(a). For Type II phantoms, pulsation was generated from a parametric biomechanical model according to a pressure waveform, the mechanical properties of the wall, and the geometry. In this model, the fluid-dynamic equations describing the haemodynamics inside the vessel are coupled with the structural equations describing the surface pulsation. The equations were solved by finite element analysis using COMSOL Multiphysics V3.4 (COMSOL Inc., Burlington, MA). Details of the modeling can be found in [35]. To the best of our knowledge, in vivo measurements of such properties close to cerebral aneurysms are not available in the literature. Therefore, literature values from arteries belonging to the Circle of Willis were set to the dome [36], [37]: a thickness of $0.050 \mathrm{~cm}$, a 
(a)

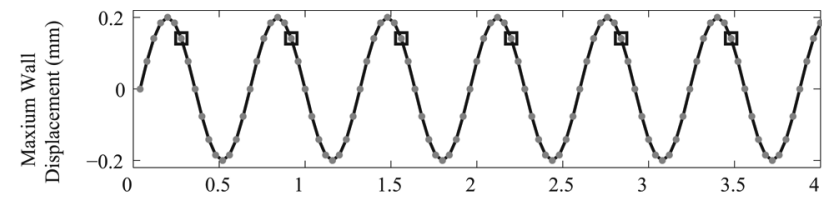

Acquisition Time (s)

(b)

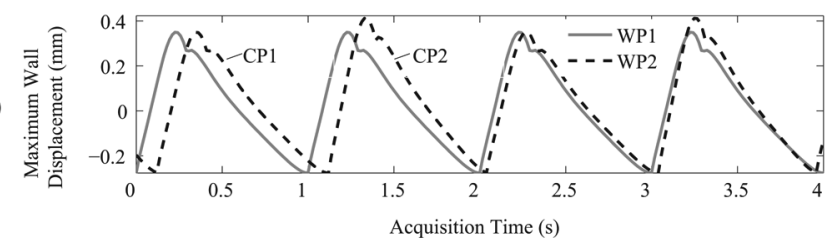

Fig. 5. Pulsation waveforms expressed as maximum wall displacement. (a) Sinusoidal waveform under ideal conditions, with pulsation amplitude range of $4 \%$ of the dome diameter for a 10-mm Type I phantom. Images are captured and repeated at the same time for each cycle, e.g., the seventh images correspond to exactly the same pulsation state (boxes). (b) Physiological waveform under: ideal conditions, WP1, and realistic conditions, WP2 (two cycle waveforms, $\mathrm{CP} 1$ and $\mathrm{CP} 2$, occurring alternatively).

tissue elastic modulus of $0.8 \mathrm{MPa}$, and a hypertensive blood pressure range of $100-170 \mathrm{mmHg}$. Bleb regions (Fig. 4) were assigned to have a slightly higher pulsatility, whereas vessel regions were set to be wall compliant. Pulsation amplitude range $(0.08-0.69 \mathrm{~mm})$ was less than $7 \%$ of the dome diameter, consistent with literature studies: $6 \%$ in [38], $0.7 \mathrm{~mm}(14 \%)$ in [37], $0.2-0.35 \mathrm{~mm}(2 \%-3 \%)$ in [39] and $0.24-0.25 \mathrm{~mm}$ $(3.8 \%-4.5 \%)$ of arterial wall motion in [40]. Periodic (WP1) and aperiodic (WP2) pulsation waveforms were considered, and an example is shown in Fig. 5(b). For WP2, two cycles, $\mathrm{CP} 1$ and $\mathrm{CP} 2$, different in amplitude and period, are combined alternatively to create an aperiodic waveform.

3) Simulated Volume and Projections: To recreate realistic 3DRA imaging, a flat panel system Allura Xper FD20 (Philips Healthcare, Best, The Netherlands) was chosen as a reference. First, a sequence of volume images was generated from the sequence of ground-truth geometries. Voxel intensities were obtained as a function of the signed distance from the voxel to the object surface, usually known as distance transform. The result is a binary image with a constant value inside the object and another value outside, but with a blurred band of $0.5 \mathrm{~mm}$ around the surface. Each image has approximately $50^{3}$ cubic voxels of $0.3 \mathrm{~mm}$ each side. Then, this volume sequence was used to simulate the original projections as described in Section II-C. The physical size of the projection images is $(160 \mathrm{~mm})^{2}$, being the X-ray source to detector distance and source to isocenter distance $1195 \mathrm{~mm}$ and $810 \mathrm{~mm}$, respectively. The rotational acquisition captures more than 100 projections uniformly distributed along a circular trajectory of over $200^{\circ}$ at a frame rate of 25-30 Hz. They were simulated with two sets of image resolutions: $1024^{2}$ and $512^{2}$ pixels.

4) Simulated Temporal Information: The ground-truth volumes and the original projections had two types of temporal relations. Under ideal conditions, the phantom pulsates periodically and is projected at exactly the same pulsation states in every cycle. In Fig. 5(a), projections are captured during a period comprising seven cardiac cycles, e.g., the seventh projection (boxes) in each cardiac cycle always has the same timestamp in the canonical cardiac cycle, and therefore reflects the same phantom pulsation state. As a result the ground-truth pulsation in the canonical cardiac cycle is known, allowing to determine the motion estimation errors. Under realistic conditions, the phantoms experience cardiac cycle variations both in pulsation amplitude and frequency, such as in Fig. 5(b). Such slight variations are likely to happen, limiting our method to recover an averaged morphodynamic behavior due to the use of the weighting scheme.

\section{B. Results}

Once an aneurysm is located, physicians routinely perform manual measurements to evaluate the lesion extent and to plan the most suitable treatment. Typically, aneurysm dome diameters and volume are calculated. Therefore, these two measures were chosen to quantify the global pulsation in our studies as well. Fig. 6 shows for both types of phantoms diameter and volume variations over time with respect to the reference volume. Three diameters are measured as the main axes dimensions of the aneurysm, viz. dome depth, length and width, as shown in Fig. 4(a). Results indicate that the estimated temporal evolution of the dome volume is more accurate than that of the diameter. Such difference can be explained by a higher sensitivity of diameters to local errors in motion estimation which are less critical in global volume estimates. Note that for Type II phantoms under realistic conditions [Fig. 6(c) and (f)], our method obtains an averaged approximation of the motion during the whole acquisition.

In fact the pulsation amplitude is expected to change at different locations on the aneurysmal wall [12], [13], indicating weaker regions, which could potentially become rupture sites. In this paper, the point-to-surface distance was used to approximate local pulsation amplitude. The geodesic active contour [41] segmentation algorithm was used to obtain the aneurysm surfaces. Fig. 7 shows for a Type I phantom with $4 \%$ pulsation of $12-\mathrm{mm}$ dome (equivalent to a pulsation range of -0.24 to $0.24 \mathrm{~mm}$ ), the color-coded estimated pulsation amplitude distribution for eight selected time points in the canonical cycle. Ground-truth color maps indicate that the bleb experiences the largest wall displacements, being in agreement with our estimates. Note that in our experiments, the maximum pulsation amplitudes are smaller than the voxel size. Nonetheless, our method was able to estimate and recover it, mainly due to the transformation properties: continuity and smoothness.

The following experiment was performed to analyze the impact of the weighting window width $\delta$, and to find a criterion of choosing the optimal $\delta$. First, with a large $\delta$, the blurring of larger pulsation variation regions is caused by the effect of averaging more projections. Second, with a smaller $\delta$, less pulsation variation is considered resulting in a more precise estimation, but the less projections are provided, the more probable the partial pulsation estimation. Results for Type II phantoms under realistic conditions using various weighting window widths are reported in Fig. 8. With $\delta \leq 10 \%$ in general our method is able to capture the pulsation. However, results with $\delta \leq 5 \%$ better recover the two-peak pulsation waveform. Note that in our case, even for $\delta=3.33 \%$ there are in average four projections per weighting window. Therefore, in practice choosing the value of $\delta$ between $5 \%-10 \%$ is a good choice, preserving the balance 

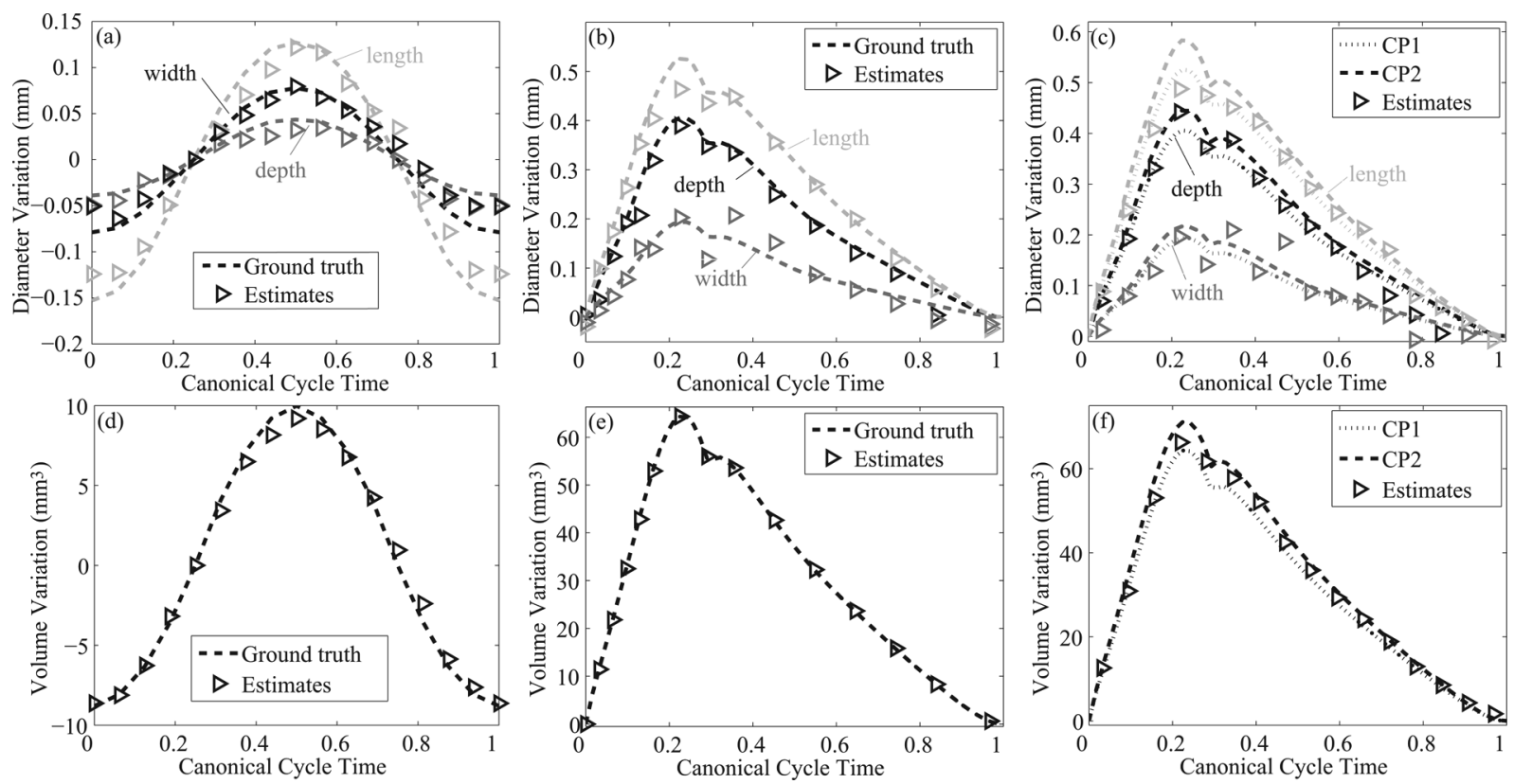

Fig. 6. Instantaneous changes in diameter (top) and volume (bottom) measured over time: (a), (d) Type I phantom. (b), (e) Type II phantom with pulsation pattern WP1. (c), (f) Type II phantom with pulsation pattern WP2, composed of two cycle waveforms CP1 and CP2, shown in Fig. 5(b).

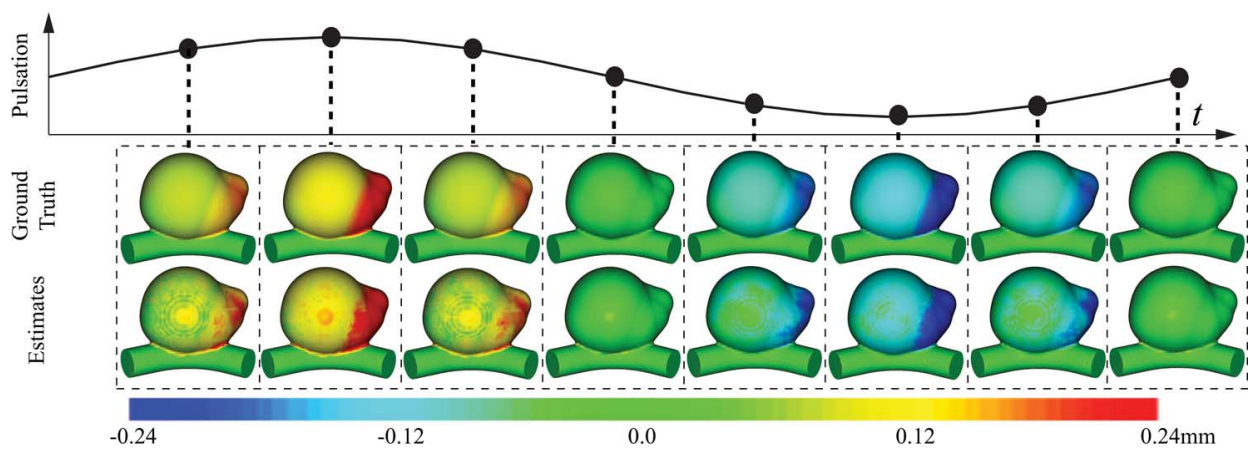

Fig. 7. Instantaneous estimated pulsation amplitude distribution at eight (dots) time points referenced to the ground truth pulsation curve. A 12-mm aneurysm with pulsation of $4 \%(0.48 \mathrm{~mm})$. Ground truth (top) is compared to the results from our method (bottom).
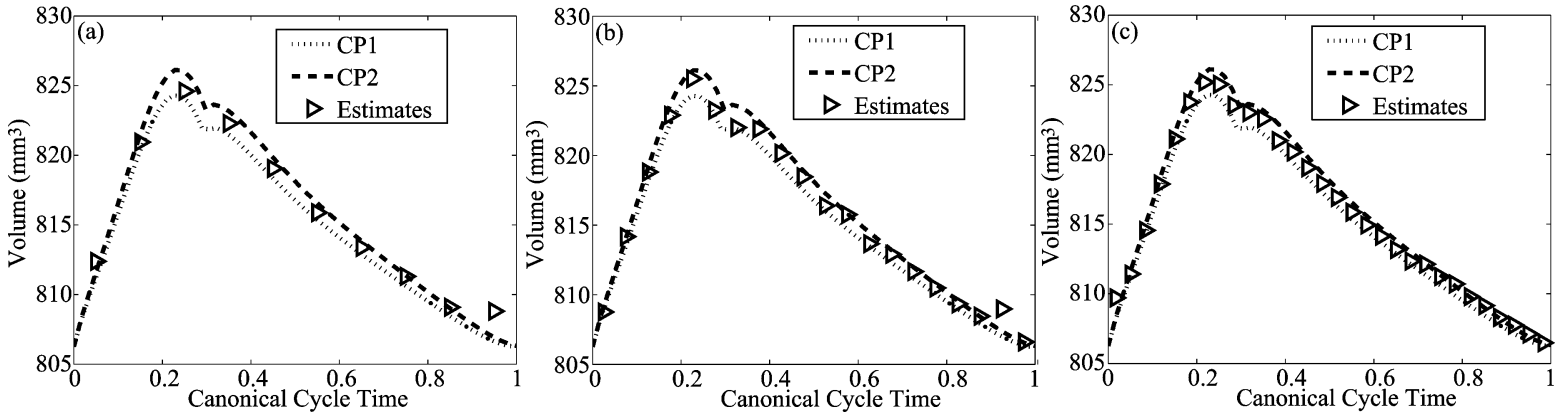

Fig. 8. Instantaneous volume measured over time for Type II phantoms under realistic conditions using weighting window width $\delta$ equal to: (a) $10 \%$, (b) $5 \%$, and (c) $3.33 \%$ of the canonical cycle.

between local pulsation variation and the number of projections available within this time interval. We chose $\delta=5 \%$ for the remaining experiments in this paper.

Although ground truth and the estimates are in good qualitative agreement, a more comprehensively quantitative compar- ison is necessary. Thus, a relative estimation error at time point $t$ in the canonical cardiac cycle is defined as

$$
\epsilon(t)=100 \times\left|\frac{m_{r}(t)-m_{g}(t)}{\hat{m}_{g}}\right|
$$




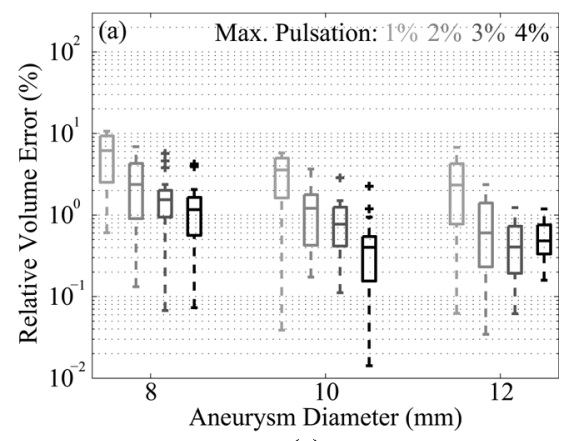

(a)

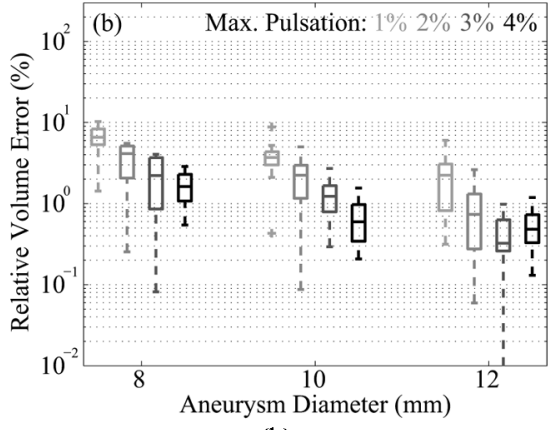

(b)

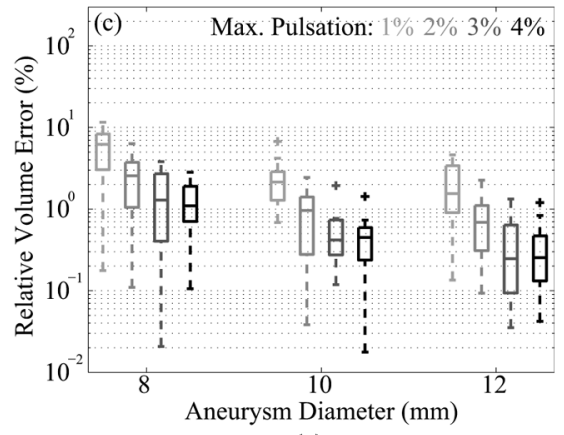

(c)

Fig. 9. Boxplots of $\epsilon$ in volume changes at 16 time points over the canonical cardiac cycle under ideal conditions, for Type I phantoms with diameter 8 , 10, and $12 \mathrm{~mm}$, and pulsation amplitude range with $1 \%, 2 \%, 3 \%$, and $4 \%$ of the dome diameter. (a) $B$-spline control points: $5^{3}$, pixel size (mm ${ }^{2}$ ): $0.31^{2}$. (b) $B$-spline control points: $8^{3}$, pixel size $\left(\mathrm{mm}^{2}\right): 0.31^{2}$. (c) $B$-spline control points: $8^{3}$, pixel size $\left(\mathrm{mm}^{2}\right): 0.16^{2}$.

where $m_{g}$ and $m_{r}$ represent the pulsation measurements (e.g., volume), $m_{g}(t)$ the ground-truth measurement at $t, m_{r}(t)$ the corresponding estimated measurement, and $\hat{m}_{g}$ the range of variation for $m_{g}(t)$ over the canonical cycle. As a result, the error $\epsilon$ expresses the estimation error as a percentage of the pulsation range.

Fig. 9 summarizes the relative volume error $\epsilon$ in boxplots [42] to represent the results for 12 cases of Type I phantoms with diameter 8,10 , and $12 \mathrm{~mm}$, each having maximum pulsation amplitudes of $1,2,3$, and $4 \%$, respectively. In all cases, our method was applied to 16 evenly sampled time points using projection image resolutions of $(0.31 \mathrm{~mm})^{2}$ or $(0.16 \mathrm{~mm})^{2}$ and a number of $B$-spline grid control points of $5^{3}$ or $8^{3}$ in a region of about $(15 \mathrm{~mm})^{3}$. In most cases, the error is less than $10 \%$ of the pulsation range. And the larger and the more pulsating is the aneurysm, the smaller the recovered relative volume error. Furthermore, Fig. 9(a) and (b) illustrate that the benefit from having more control points is not significant. A possible explanation is that, as the fine grid increases dramatically the number of optimization parameters, the method becomes more sensitive to the noise present in the data. In our studies, the optimization was not able to handle more than 10 control points per axis, 6591 estimated parameters in total. On the other hand, because of the sparse derivative matrix described in the Appendix, the computational time does not increase significantly with a larger number of control points. Fig. 9(b) and (c) suggest that the resolution of the original and simulated projections can be reduced without significantly degrading the accuracy of the final estimation. Similar findings have been mentioned in [43]. For this reason, for the remaining experiments with digital phantoms, projection image resolution was fixed to $(0.31 \mathrm{~mm})^{2}$ and a number of $B$-spline grid control points of $8^{3}$ in a region of about $(15 \mathrm{~mm})^{3}$.

\section{Discussion}

As the expected cerebral aneurysmal motion range is very small, e.g., subvoxel, accurately estimating such dynamic morphology is challenging. For this reason, in this section we evaluate the impact of different factors that might affect the motion estimation accuracy.

1) Effects of Head Movement: Although patients are usually sedated during the acquisition, slight head movements might happen. In our work, head movements were simulated when acquiring each original projection, by adding a 4-degree-of-freedom rigid movement, i.e., three translations and a rotation along the gantry trajectory. In the first set of experiments, for each original projection, randomly generated translations within the range of the maximum pulsation amplitude and a rotation within $0.1^{\circ}$ were added to the reference volume before projecting it. Fig. 10(a) shows the relative volume errors using the same set of phantoms as used in Fig. 9. Results suggest that although slight head movements degrade the performance, the accuracy of the method is still satisfactory as most errors are bounded and below 10\%. In [44], head movements are observed to be less than $0.5 \mathrm{~mm}$ during the first $5 \mathrm{~min}$. In the case of a 3DRA acquisition, patient is positioned in the same manner but for a shorter time, and in a second set of movement simulations, the randomly generated translations and rotation ranges were set to be $0.25 \mathrm{~mm}$ and $0.1^{\circ}$, respectively. As shown in Fig. 10(b), the error raises dramatically under this condition, since the introduced head movements can be even larger than the pulsation. Therefore it is necessary to guarantee immobility of the patient.

2) Effects of Scattering and Noise: X-ray projections are measured from photon counts, which are always degraded by scattered photons, and quantum noise is dominated by the Poisson effect. In order to study their influence, scattering and noise are simulated and added to the scattering-noise-free projections. Scattering is modeled using convolution filtering to blur the projections. A 2D Gaussian function with a full-width at half-maximum (FWHM) of 75 pixels (i.e., $23.4 \mathrm{~mm}$ ) and a scatter-to-primary ratio (SPR) of $10 \%$ was convolved with the projections [45]. Then quantum noise is simulated using a Box-Muller approximation of a Gaussian distribution, which is representative as the number of photons emitted is sufficiently high [46]. The resultant Gaussian noise has a zero mean and standard deviation of $2.5 \%$ of the maximum value of the scattered image signal [47]. In practice, the SPR and the amount of noise might be even larger. Results are shown in Fig. 10(c). The performance is slightly degraded by scattering and noise, but the error is still less than $10 \%$ in most of the cases. Cases with higher pulsation and larger size are less affected, thus providing a similar error range as obtained from scattering-noise-free experiments shown in Fig. 9(b). 

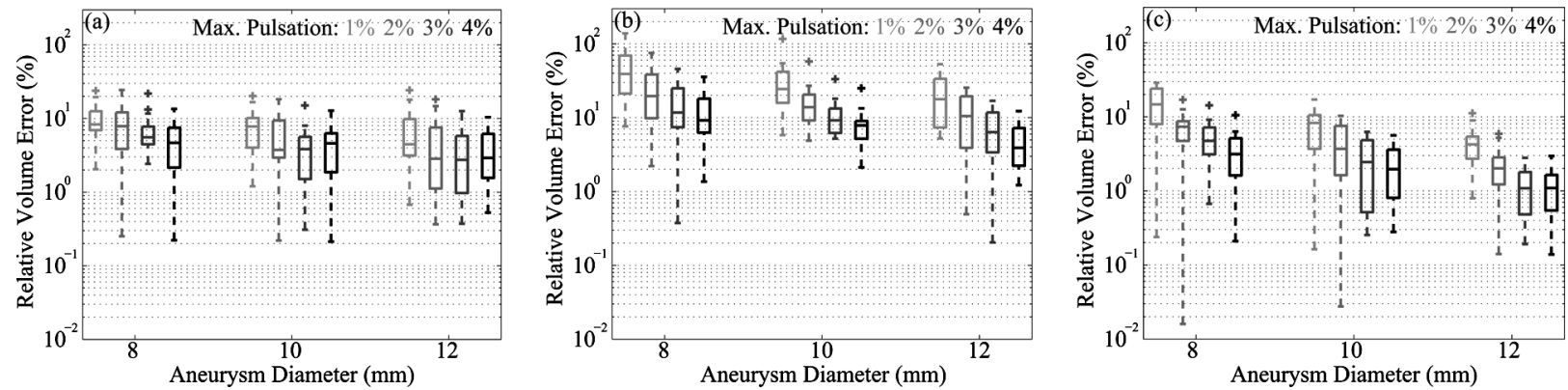

Fig. 10. Boxplots of $\epsilon$ in volume changes at 16 time points over the canonical cardiac cycle for the same phantoms in Fig. 9 using: (a) randomly added translations and rotation, within the maximum pulsation amplitude and $0.1^{\circ}$, (b) randomly added translations and rotation, within $0.25 \mathrm{~mm}$ and $0.1^{\circ}$, and $(\mathrm{c}) \mathrm{SPR}=10 \%$ plus a Gaussian noise with zero mean and standard deviation of $2.5 \%$ of the maximum value of the scattered image signal.
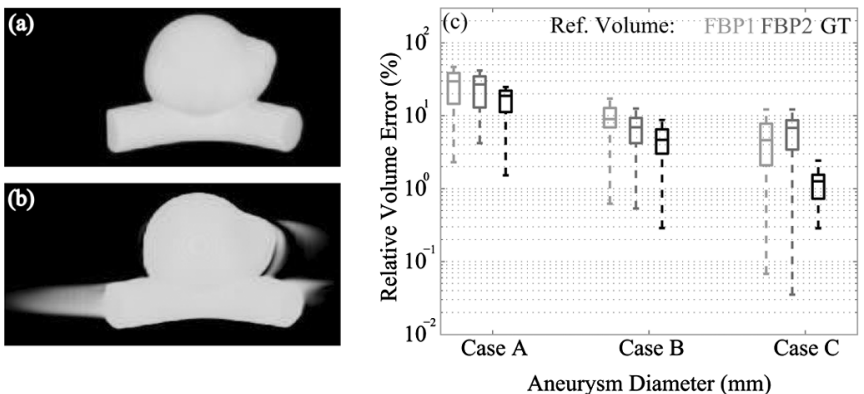

Fig. 11. (a) The ground-truth volume at the averaged state. (b) Volume reconstructed using FBP reconstruction algorithm, showing some undesired artifacts. (c) Boxplots of $\epsilon$ in volume changes at 16 time points over the canonical cardiac cycle using three types of reference volumes (FBP1, FBP2, and GT). Case A, diameter $8 \mathrm{~mm}$ with $1 \%$ pulsation; Case B, $10 \mathrm{~mm}$ with $3 \%$, and Case C, $12 \mathrm{~mm}$ with $3 \%$.

3) Effects of Reference Volume Selection: In clinical practice, although 3DRA images are filtered to reduce the presence of reconstruction artifacts, they are not completely removed, as shown in Fig. 11. Meanwhile, when reconstructed from the projections with changing morphology, the reference volume is expected to represent a smoothly averaged state. Three sets of experiments were performed to study the possible influence by different choices of the reference volume. In the first set, the ground-truth volume at the averaged state was used; in the second set, the reference volume was reconstructed from the simulated projections of the pulsating phantom, using the FBP algorithm [19]; and in the third set, the reference volume was obtained the same way as the second set, but the phantom was not pulsating. We denote these three sets as GT, FBP1, and FBP2, respectively, for the simplification of explanation. Three Type I phantom cases were tested: diameter $8 \mathrm{~mm}$ with $1 \%$ pulsation (Case A), $10 \mathrm{~mm}$ with 3\% pulsation (Case B), and $12 \mathrm{~mm}$ with $3 \%$ pulsation (Case $\mathrm{C}$ ). The estimated relative volume errors are shown in Fig. 11(c). Larger errors were obtained when using reference volumes from the FBP reconstruction, whereas results were not substantially different by using FBP1 or FBP2. However, the error variations introduced by different sizes and pulsation amplitudes are not significant, either. This suggests that the artifacts present in the FBP reconstructions introduce a nonnegligible amount of error in our estimation.

4) Effects of Adding Realistic Background: Besides the studied aneurysm, patient images also contain other attenuated
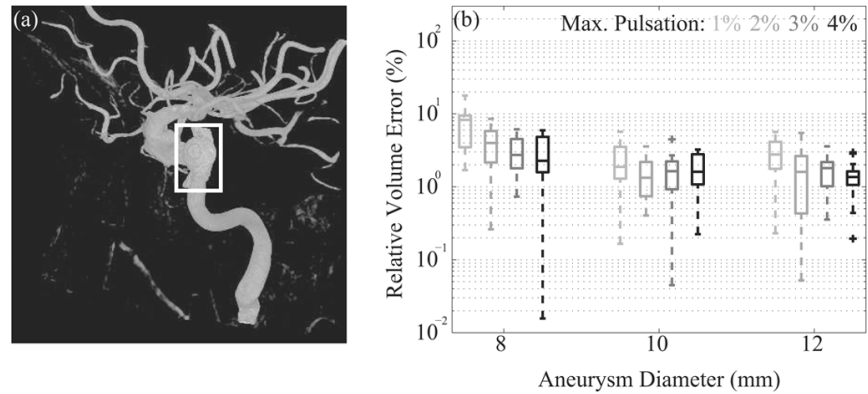

Fig. 12. (a) A digital phantom blended with realistic background (white frame indicating the aneurysm phantom). (b) Boxplots of $\epsilon$ in volume changes at 16 time points over the canonical cardiac cycle for such dataset.

vessels, air, bones, and soft tissues. In order to study their influence, we have blended Type I digital pulsating phantoms into a real patient dataset. Once each phantom was placed in the patient image, the voxels corresponding to aneurysm and vessel were set to a representative vessel intensity value. An example of the blended projections and volumes is shown in Fig. 12(a). Fig. 12(b) shows the relative volume errors using such simulation dataset. Compared with Fig. 9(b), the errors are in general larger, and the error variations introduced by sizes and pulsation amplitudes are smaller. Nonetheless, the results indicate that adding the realistic background into the digital phantom experiments does not significantly degrade the performance, as the errors in most cases are below $10 \%$.

5) Effects of Angular Resolution of the Projections: It is found that the accuracy and robustness of a 3D-to-multiple-2D image registration method do not depend solely on the method itself but also on the number of projections [48]. We have investigated this factor by downsampling the number of original projections and using only the subsampled set. The downsampling factors (df) used in our experiments were 2 and 3. As this factor indeed determines the density of the projections along the rotational gantry trajectory, we refer to it as angular resolution of the projections. Fig. 13 summarizes the relative volume errors on these experiments for 12 cases of Type I phantoms. As can be seen from Fig. 13(a) and (b), reducing the number of projections by a factor of 2 slightly increases the error. But it remains below $10 \%$ in most of the cases. Fig. 13(c) shows that the errors are mostly below $20 \%$ when reducing the number of projections by a factor of 3 . These experiments indicate that the 

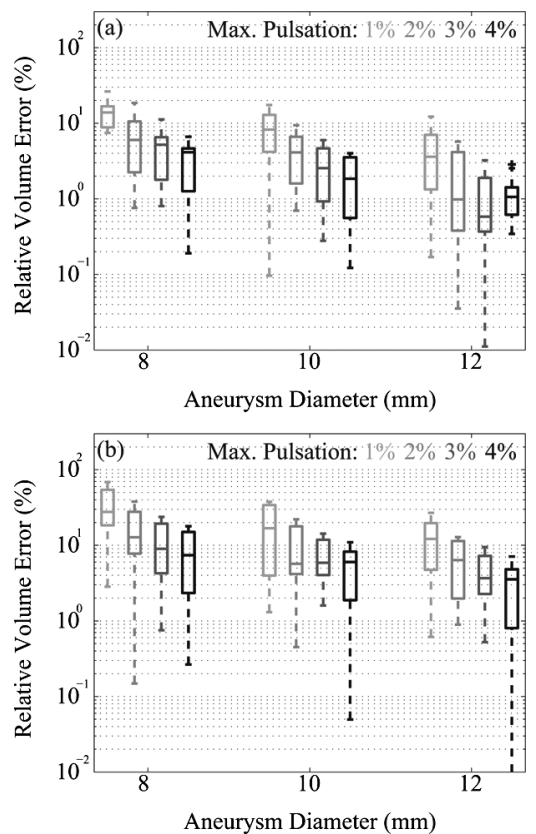

Fig. 13. Boxplots of $\epsilon$ in volume changes at 16 time points over the canonical cardiac cycle for the same phantoms as used in Fig. 9 but with the downsampling factor: (a) $\mathrm{df}=2$; (b) $\mathrm{df}=3$.

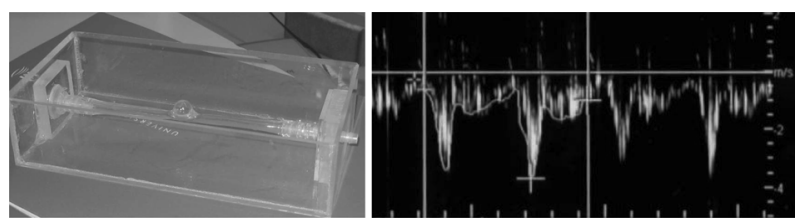

Fig. 14. The silicone side-wall aneurysm phantom (left) and the Doppler ultrasound velocity waveform at the parent vessel inlet (right).

performance increases when the angular resolution of the projections is higher.

\section{PHySICAL PHANTOM EXPERIMENTS}

The method has also been tested in a real clinical environment, using a nonrigid silicone phantom under pulsatile flow conditions. One obstacle is to evaluate the accuracy due to the absence of ground truth information. Results are evaluated firstly by visual inspection comparing the simulated projections from the estimated volumes to the corresponding original projections. And secondly, they are compared to Doppler ultrasound flow waveforms obtained in the experimental setup.

\section{A. Data Acquisition}

A silicone side-wall aneurysm phantom was used (Fig. 14). It has a 10-mm-diameter spherical aneurysm dome and a 4-mm-diameter cylindrical vessel. The phantom was water-filled and connected to a customized pulsatile pump, a continuous flow pump (Elastrat, Geneva, Switzerland), and a liquid tank to create a continuous and pulsatile fluid circulation. The box containing the phantom was also filled with water to simulate realistic X-ray attenuation. The pulsatile pump produced a human-like pulsatile flow, as shown in Fig. 14. As a result, the phantom presented visible wall motion.
TABLE II

DEsign PARAMETERS For Physical Phantom STUdies

\begin{tabular}{lc}
\hline Parameters & Values \\
\hline Dome shape & Spherical \\
Dome diameter $(\mathrm{mm})$ & 10 \\
Vessel shape & Cylindrical \\
Vessel diameter $(\mathrm{mm})$ & 4 \\
& \\
Pulsation waveform & Pseudo Physiological \\
Pulsation frequency (bpm) & 90 \\
Pulsation amplitude range $(\mathrm{mm})$ & unknown \\
& \\
Frame rate $(\mathrm{Hz})$ & 30 \\
Projection spacing $\left(\mathrm{mm}^{2}\right)$ & $0.155^{2}$ \\
Volume spacing $\left(\mathrm{mm}^{3}\right)$ & $0.295^{3}$ \\
\hline
\end{tabular}

Experimental data were acquired using a flat panel system Allura Xper FD20 (Philips Healthcare, Best, The Netherlands). In total 121 rotational projection images were captured with a frame rate of $30 \mathrm{~Hz}$, over $200^{\circ}$ along the gantry trajectory. Constant injection of contrast agent Iomeron 400 (Bracco Imaging $\mathrm{SpA}$, Milan, Italy) was performed during the acquisition at a rate of $3 \mathrm{~mL} / \mathrm{s}$. X-ray source and detector positions were recorded for each projection, allowing the spatial relationship between the reconstructed reference volume and each projection to be known. The reference volume has $256^{3}$ cubic voxels of $0.295 \mathrm{~mm}$ each side. The original projections have a dimension of $1024^{2}$ with pixel size of $(0.155 \mathrm{~mm})^{2}$. Table II summarizes the parameters of the phantom geometry, pulsation, and imaging conditions.

\section{B. Experiments and Results}

A number of $B$-spline control points $(8 \times 8 \times 8)$ were set to a region of about $(11 \mathrm{~mm})^{3}$ in size, containing the aneurysm dome. The pulsatile pump signal frequency was set to $90 \mathrm{bpm}$, and the acquisition frame rate was $30 \mathrm{~Hz}$. Thus during each pulsation cycle, projections were acquired at the same 20 instants of time within the cycle, which means the experiments were performed under ideal conditions. This allows validating the results at each ground-truth pulsation state by comparing the projections of the estimated $3 \mathrm{D}$ volumes to the corresponding original ones.

Fig. 15(a) and (b) shows from two viewpoints the X-ray projections of one acquisition under pulsatile flow conditions. In Fig. 15(b) and (e), the regions of checkerboard images presenting the aneurysm dome are shown, where each checkerboard image is composed of the X-ray projection and its corresponding simulated projection of the reference volume. A clear mismatch on both dome and vessel boundaries can be observed. Whereas in Fig. 15(c) and (f), similar checkerboard images are shown from the estimated volumes. Such checkerboard comparison shows that our method was able to correct the misalignment caused by pulsation.

Fig. 16(a) shows the estimated volume variations. Similar results were obtained using two downsampling factors: $\mathrm{df}=1,2$. This is in agreement with the digital phantom experiments, as shown in Section III-C5. Differences at the peak should be explained by the lack of information to constrain the deformation in certain directions. Along with the volume variations, the Doppler ultrasound velocity waveform measured at the aneurysm parent vessel inlet was also shown. 

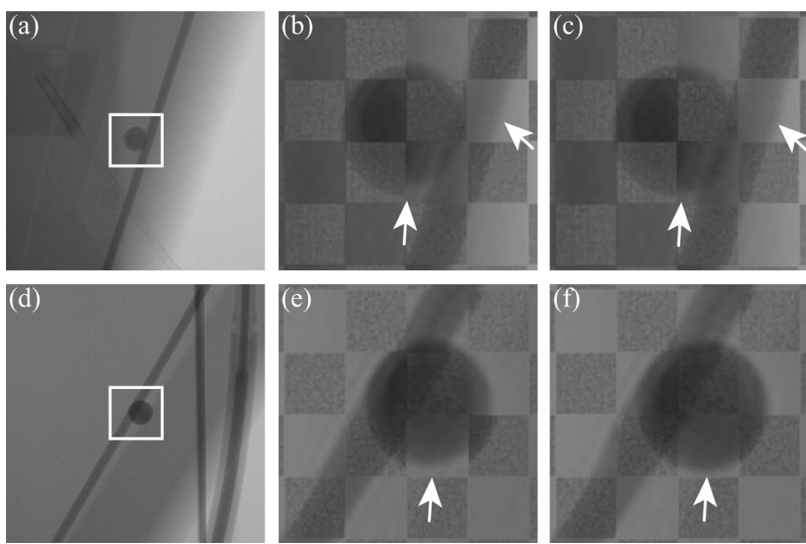

Fig. 15. (a), (d) X-ray projection images, each with a white frame indicating the regions shown in (b), (c) and (e), (f), respectively. Checkerboard comparisons of the original and simulated projections: (b), (e) before applying our method and (c), (f) afterwards

(a)

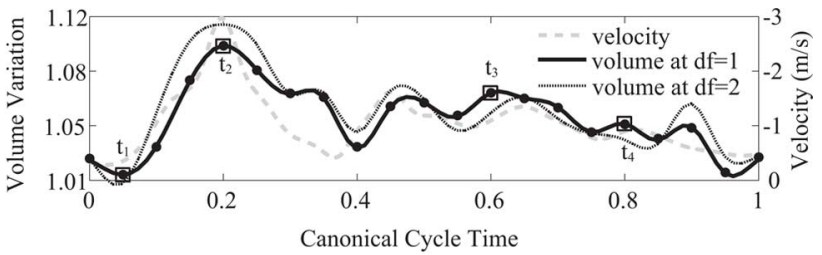

(b)

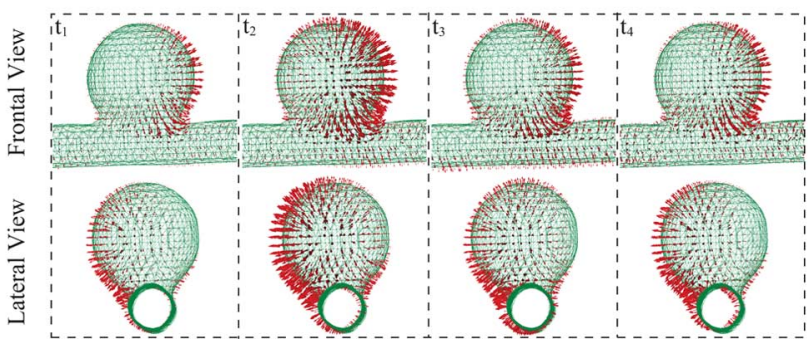

Fig. 16. (a) The obtained volume variations with two downsampling factors df $=1,2$, along with the Doppler ultrasound velocity waveform. (b) Four (boxes) out of 20 (dots) estimated time points presenting the instantaneous wall displacements with respect to the reference geometry.

It should be noted that, apart from the phase delay due to the coupling between the fluid flow and wall displacement [40], the velocity was not synchronized with the data acquisition, thus a waveform shift possibly exists. Nonetheless, the volume variation waveform qualitatively presents a similar pattern to the velocity waveform, which is in agreement with the findings in [38], [40]. An advantage of measuring volume is that, the global movements are not reflected in volume changes, resulting in only the deformation of the aneurysm phantom. Fig. 16(b) shows at four out of 20 estimated time points the instantaneous wall displacements with respect to the reference geometry. It can be observed that wall displacements are larger at the flow impingement area. In addition to dome expansion and contraction, the phantom experiences a tilting movement towards the flow direction as well.

Fig. 17 plots for each point on the segmented surface of the reference volume its color-coded pulsation amplitude range over the cardiac cycle, together with its histogram of the deformed region. Only a small region has higher pulsatility, which can be observed from the color-coded map. Note that the
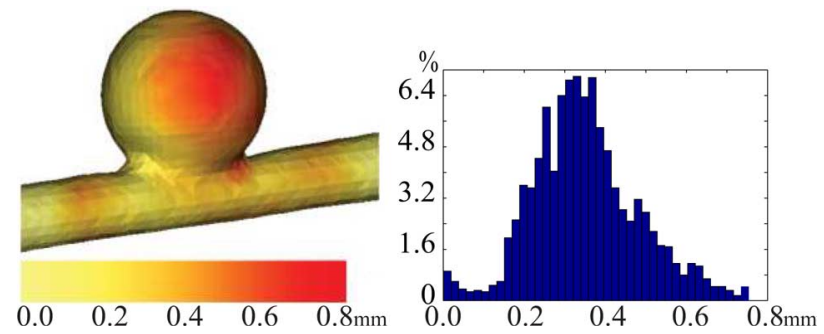

Fig. 17. Color-coded displacement range over the cardiac cycle for each point on the surface, and the histogram of the displacement range.

rest of the deformed region has an averaged pulsation range of around $0.3 \mathrm{~mm}$, part of which could be accounted for a global rigid movement.

\section{DISCUSSION}

To validate the proposed methodology, experiments with digital and physical phantoms were performed. The phantoms presented aneurysms with pulsation ranges consistent with literature values. The imaging conditions matched what standard 3DRA imaging suites offer nowadays.

Experiments with digital phantoms were performed under controlled conditions, allowing to evaluate the sensitivity of our method to various factors. Results under ideal conditions showed estimation errors below $10 \%$ of the maximum pulsation, which in general presented subvoxel wall displacements not exceeding two voxels. In terms of algorithm parameters such as control grid size and weighting window width, similar results have been obtained. In terms of imaging conditions, different projection image resolutions provided similar estimation results; scattering and quantum noise degraded slightly the performance (maximum error of $20 \%$ ) for smaller pulsation and aneurysm size cases (e.g., $8 \mathrm{~mm}$ with $1 \%$ maximum pulsation); FBP reconstruction artifacts decreased the performance, in general below the $10 \%$ except for the smaller pulsation aneurysms; angular resolution of the projection data is preferred to be higher. In case of disturbances from the imaged object such as head movement, estimation error increased to $20 \%$ with small perturbations. The presence of background objects raised slightly the estimation errors.

Experiments with the physical phantom allowed to demonstrate the feasibility of pulsation estimation under clinical conditions using anthropomorphic aneurysm replicas. The mismatches due to pulsation between the original projections and the simulated projections of the reference volume were corrected in the estimated volumes. In addition, the volume variation waveform presented a similar pattern compared to measured Doppler ultrasound velocity waveforms. Further validation could consider studying the mechanical relationship between the pressure/velocity and dynamic aneurysmal wall compliance.

A challenge for the method to be applied in large population studies and routine clinical practice is the computational cost. The high computational effort is partially compensated for by the current implementation, as mentioned in the Appendix. It takes on average 10-15 min for one selected time point using a Pentium 4-3 GHz PC, which requires further speed-up. The 
computing bottleneck is generating the simulated projections and computing the pixel-wise similarity metric. This could be potentially solved by either implementing them into hardware or using parallel computing.

Cerebral aneurysms could be more irregular in shape than the ones used in the experiments and little is known about their exact pulsation pattern and behavior at rupture. Therefore, the anatomical structure and pulsation waveforms used in these experiments might represent a simplified approximation of reality. Quantification of the accuracy of our method on patient data and large population studies must be performed in the future. Methodological improvements under realistic acquisition settings might include the development of density-variable $B$-spline grids so that local deformation in small blebs can be optimally captured.

\section{CONCLUSION}

The objective of this paper was to develop a methodology able to characterize patient-specific pulsatility of cerebral aneurysms over the cardiac cycle using 3DRA acquisitions. As a result, a registration-based technique has been proposed to recover motion from a reference $3 \mathrm{D}$ volumetric image and a set of $2 \mathrm{D}$ rotational projections synchronously acquired with a cyclic physiological signal. A number of experiments with digital and physical phantoms did illustrate the feasibility of estimating aneurysmal motion during the cardiac cycle.

Advantages of the presented technique can be detailed as follows: first, it does not require exposing patients to additional radiation as it uses the standard acquisition performed during an endovascular treatment. Second, compared to traditional algorithms of reconstruction from projections, it preserves the high spatial resolution of the 3DRA volume in spite of using a sparse set of projections because of the continuity and smoothness of the deformation model. In addition, its general formulation could be used to estimate motion of other organs.

\section{APPENDIX \\ GRADIENT OF THE REGISTRATION METRIC}

The gradient of the metric in (3) with respect to the parameters $\boldsymbol{\omega}$ can be expressed as

$$
\frac{\partial}{\partial \boldsymbol{\omega}} M(\boldsymbol{\omega} ; t)=\sum_{k} \mu\left(t, t_{k}\right) \frac{\partial}{\partial \boldsymbol{\omega}} D\left(\tilde{I}_{k}(\boldsymbol{\omega}), I_{k}\right)
$$

For the mutual information metric (4)

$$
\frac{\partial}{\partial \boldsymbol{\omega}} D\left(\tilde{I}_{k}(\boldsymbol{\omega}), I_{k}\right)=\frac{\partial D\left(\tilde{I}_{k}(\boldsymbol{\omega}), I_{k}\right)}{\partial p(u, v)} \cdot \frac{\partial p(u, v)}{\partial \boldsymbol{\omega}}
$$

where

$$
\begin{aligned}
\frac{\partial p(u, v)}{\partial \boldsymbol{\omega}}=\frac{1}{\Delta \tilde{h} \cdot N_{k}} \sum_{\mathbf{x} \in \mathcal{R}_{k}} \beta^{(0)}\left(u-\frac{I_{k}(\mathbf{x})-i_{k}^{\circ}}{\Delta h}\right) \\
\left.\cdot \frac{\partial \beta^{(3)}(\hat{v})}{\partial \hat{v}}\right|_{\hat{v}=v-\frac{\tilde{I}_{k}(\mathbf{x} ; \boldsymbol{\omega})-\tilde{i}_{k}^{\circ}}{\Delta \hat{h}} \cdot\left(-\frac{\partial \tilde{I}_{k}(\mathbf{x} ; \boldsymbol{\omega})}{\partial \boldsymbol{\omega}}\right)}
\end{aligned}
$$

$N_{k}$ is the number of pixels in the valid ray region $\mathcal{R}_{k}$, and

$$
\frac{\partial}{\partial \boldsymbol{\omega}} \tilde{I}_{k}(\mathbf{x} ; \boldsymbol{\omega})=\alpha(k, \mathbf{x}) \times \sum_{\ell} \nabla V\left(\mathcal{T}\left(\mathbf{p}_{\ell} ; \boldsymbol{\omega}\right)\right) \cdot \frac{\partial}{\partial \boldsymbol{\omega}} \mathcal{T}\left(\mathbf{p}_{\ell} ; \boldsymbol{\omega}\right)
$$

where $\nabla V$ denotes the spatial gradient of the volume.

Observing that, for $B$-spline transformations the Jacobian matrix $\partial \mathcal{T}\left(\mathbf{p}_{\ell} ; \boldsymbol{\omega}\right) / \partial \boldsymbol{\omega}$ is a sparse matrix related to the order of the $B$-spline function, allowing to significantly speed up the computation. Furthermore, the evaluation of the metric and its gradient can be speeded up by just computing it within the valid ray region $\mathcal{R}_{k}$ for each projection.

\section{ACKNOWLEDGMENT}

The authors would like to thank Dr. D. Rüfenacht and L. Augsburger from Geneva University Hospitals (HUG) for providing the aneurysm silicone phantom and the pulsatile pump, R. Hermans from Philips Healthcare (Best, The Netherlands) for the FBP reconstruction software, Dr. J. Blasco, Dr. J. Macho, and Dr. E. Vivas for the 3DRA acquisitions of the physical phantom, S. Balocco for building the digital phantoms using the COMSOL software, and H. Bogunović for the segmentation software.

\section{REFERENCES}

[1] J. Brisman, J. Song, and D. Newell, "Medical progress: Cerebral aneurysms," New Eng. J. Med., vol. 355, no. 9, pp. 928-939, 2006.

[2] J. Huang and J. M. van Gelder, "The probability of sudden death from rupture of intracranial aneurysms: A meta-analysis," J. Neurosurg., vol. 51, no. 5, pp. 1101-1105, 2002.

[3] G. Hademenos, T. Masssoud, F. Turjman, and J. Sayre, "Anatomical and morphological factors correlating with rupture of intracranial aneurysms in patients referred for endovascular treatment," Neuroradiology, vol. 40, pp. 755-760, 1988.

[4] R. Millán, L. Dempere-Marco, J. Pozo, J. Cebral, and A. Frangi, "Morphological characterization of intracranial aneurysms using 3D moment invariants," IEEE Trans. Med. Imag., vol. 26, no. 9, pp. 1270-1282, Sep. 2007.

[5] M. Raghavan, B. Ma, and R. Harbaugh, "Quantified aneurysm shape and rupture risk," J. Neurosurg., vol. 102, pp. 355-362, 2005.

[6] F. Meyer, J. H. III, and S. Riederer, "Pulsatile increases in aneurysm size determined by cine phase-contrast MR angiography," J. Neurosurg., vol. 78, no. 6, pp. 879-883, 1993.

[7] J. Wardlaw, J. Cannon, P. Statham, and R. Price, "Does the size of intracranial aneurysms change with intracranial pressure? Observations based on color "power" transcranial Doppler ultrasound," J. Neurosurg., vol. 88, no. 5, pp. 846-850, 1998.

[8] J. Wardlaw and J. Cannon, "Color transcranial "power" Doppler ultrasound of intracranial aneurysms," J. Neurosurg., vol. 84, no. 3, pp. 459-461, 1996.

[9] M. Hayakawa, K. Katada, H. Anno, S. Imizu, J. Hayashi, K. Irie, M. Negoro, Y. Kato, T. Kanno, and H. Sano, "CT angiography with electrocardiographically gated reconstruction for visualizing pulsation of intracranial aneurysms: Identification of aneurysmal protuberance presumably associated with wall thinning," Am. J. Neuroradiol., vol. 26, pp. 1366-1369, 2005.

[10] F. Ishida, H. Ogawa, T. Simizu, T. Kojima, and W. Taki, "Visualizing the dynamics of cerebral aneurysms with four-dimensional computed tomographic angiography," Neurosurgery, vol. 57, no. 3, pp. 460-471, 2005.

[11] Y. Kato, M. Hayakawa, H. Sano, M. Sunil, S. Imizu, M. Yoneda, S. Watanabe, M. Abe, and T. Kanno, "Prediction of impending rupture in aneurysms using 4D-CTA: Histopathological verification of a real-time minimally invasive tool in unruptured aneurysms," Minim. Invasive Neurosurg., vol. 47, pp. 131-135, 2004.

[12] L. Dempere-Marco, E. Oubel, M. Castro, C. Putman, A. Frangi, and J. Cebral, "CFD analysis incorporating the influence of wall motion: Application to intracranial aneurysms," in Proc. Int. Conf. Med. Image Comput. Computer-Assisted Intervention (MICCAI'06), 2006, pp. 438-445. 
[13] E. Oubel, M. DeCraene, C. Putman, J. Cebral, and A. Frangi, "Analysis of intracranial aneurysm wall motion and its effects on hemodynamic patterns," in Proc. SPIE Conf. Med. Imag.: Physiol., Function, Structure Med. Images, 2007, vol. 6511, no. 65112A.

[14] S. Bonnet, A. Koenig, S. Roux, P. Hugonnard, R. Guillemaud, and P. Grangeat, "Dynamic X-ray computed tomography," Proc. IEEE, vol. 91, no. 10, pp. 1574-1587, Oct. 2003.

[15] B. Movassaghi, M. Grass, D. Schaefer, V. Rasche, O. Wink, G. Schoonenberg, J. Chen, J. Garcia, B. Groves, J. Messenger, and J. Carroll, "4D coronary artery reconstruction based on retrospectively gated rotational angiography: First in-human results," in Proc. SPIE Conf. Med. Imag.: Visualizat. and Image-Guide Procedures, 2007, vol. 6509, no. $65090 \mathrm{P}$.

[16] V. Rasche, B. Movassaghi, M. Grass, D. Schäfer, H. Kühl, R. Günther, and A. Bücker, "Three-dimensional X-ray coronary angiography in the porcine model: A feasibility study," Acad. Radiol., vol. 13, no. 5, pp. 644-651, 2006.

[17] D. Schäfer, J. Börgert, V. Rasche, and M. Grass, "Motion-compensated and gated cone beam filtered back-projection for 3-D rotational X-ray angiography," IEEE Trans. Med. Imag., vol. 25, no. 7, pp. 898-906, Jul. 2006.

[18] L. Feldkamp, L. Davis, and J. Kress, "Practical cone beam algorithms," J. Opt. Soc. Am. A, vol. 6, pp. 612-619, 1984.

[19] M. Grass, R. Koppe, E. Klotz, R. Proksa, M. Kuhn, H. Aerts, J. Op de Beek, and R. Kemkers, "3D reconstruction of high contrast objects using C-arm image intensifier projection data," Comput. Med. Imag. Graph., vol. 23, pp. 311-321, 1999.

[20] C. Blondel, G. Malandain, R. Vaillant, and N. Ayache, "4D deformation field of coronary arteries from monoplane rotational X-ray angiography," in Proc. CARS, Computer Assisted Radiology and Surgery, 2003, vol. 1256, pp. 1073-1078.

[21] C. Blondel, G. Malandain, R. Vaillant, and N. Ayache, "Reconstruction of coronary arteries from a single rotational X-ray projection sequence," IEEE Trans. Med. Imag., vol. 25, no. 5, pp. 653-663, 2006.

[22] R. Zeng, J. Fessler, and J. Balter, "Estimating 3-D respiratory motion from orbiting views by tomographic image registration," IEEE Trans. Med. Imag., vol. 26, no. 2, pp. 153-163, Feb. 2007.

[23] E. Hansis, D. Schäfer, O. Dössel, and M. Grass, "Projection-based motion compensation for gated coronary artery reconstruction from rotational x-ray angiograms," Phys. Med. Biol., vol. 53, no. 14, pp. 3807-3820, 2008.

[24] B. Perrenot, R. Vaillant, R. Prost, G. Finet, P. Douek, and F. Peyrin, "Motion correction for coronary stent reconstruction from rotational X-ray projection sequences," IEEE Trans. Med. Imag., vol. 26, no. 10, pp. 1412-1423, Oct. 2007.

[25] C. Zhang, M.-C. Villa-Uriol, M. D. Craene, J. Pozo, and A. Frangi, "Time-resolved 3D rotational angiography reconstruction: Towards cerebral aneurysm pulsatile analysis," Int. J. Comput. Assist. Radiol. Surg., pp. S36-S55, 2008.

[26] J. Kybic and M. Unser, "Fast parametric elastic image registration," IEEE Trans. Signal. Process., vol. 12, no. 11, pp. 1427-1442, Nov. 2003.

[27] D. Rueckert, L. Sonoda, C. Hayes, D. Hill, M. Leach, and D. Hawkes, "Non-rigid registration using free-form deformations: Application to breast MR images," IEEE Trans. Med. Imag., vol. 18, no. 8, pp. 712-721, Aug. 1999.

[28] F. Jacobs, E. Sunderman, B. Sutter, M. Christiaens, and I. Lemahieu, "A fast algorithm to calculate the exact radiological path through a pixel or voxel space," J. Comput. Inf. Tech., vol. 6, pp. 89-94, 1998.

[29] R. Siddon, "Fast calculation of the exact radiological path for a threedimensional CT array," Med. Phys., vol. 12, no. 2, pp. 252-255, 1985.
[30] J. Hipwell, G. Penney, R. McLaughlin, K. Rhode, P. Summers, T. Cox, J. Byrne, J. Noble, and D. Hawkes, "Intensity-based 2D-3D registration of cerebral angiograms," IEEE Trans. Med. Imag., vol. 22, no. 11, pp. 1417-1426, Nov. 2003.

[31] D. Mattes, D. Haynor, H. Vesselle, T. Lewellen, and W. Eubank, "PET-CT image registration in the chest using free-form deformation," IEEE Trans. Med. Imag., vol. 22, no. 1, pp. 120-128, Jan. 2003.

[32] P. Thevenaz and M. Unser, "Spline pyramids for inter-modal image registration using mutual information," in Proc. SPIE Conf. Wavelet Appl. Signal Image Process. V, 1997, vol. 3169, pp. 236-247.

[33] R. Manzke, M. Grass, and D. Hawkes, "Artifact analysis and reconstruction improvement in helical cardiac cone beam CT," IEEE Trans. Med. Imag., vol. 23, no. 9, pp. 1150-1164, Sep. 2004.

[34] C. Zhu, R. Byrd, and J. Nocedal, "L-BFGS-B: Algorithm 778: L-BFGS-B, FORTRAN routines for large scale bound constrained optimization," ACM Trans. Math. Software, vol. 23, no. 4, pp. 550-560, 1997.

[35] S. Balocco, O. Camara, and A. Frangi, "Towards regional elastography of intracranial aneurysms," in Proc. Int. Conf. Med. Image Comput. Computer-Assisted Intervention (MICCAI'08), 2008, pp. 131-138.

[36] J. Alastruey, K. Parker, J. Peiró, S. Byrd, and S. Sherwin, "Modelling the Circle of Willis to assess the effects of anatomical variations and occlusions on cerebral flows," J. Biomech., vol. 40, no. 8, pp. 1794-1805, 2007.

[37] R. Torii, M. Oshima, T. Kobayashi, K. Takagi, and T. Tezduyar, "Fluidstructure interaction modeling of aneurysmal conditions with high and normal blood pressures," Comput. Mech., vol. 38, pp. 482-490, 2006.

[38] M. Löw, K. Perktold, and R. Raunig, "Hemodynamics in rigid and distensible saccular aneurysms: A numerical study of pulsatile flow characteristics," Biorheology, vol. 30, pp. 287-298, 1993.

[39] A. Valencia and F. Solis, "Blood flow dynamics and arterial wall interaction in a saccular aneurysm model of basilar artery," Comput. Struct., vol. 84, pp. 1326-1337, 2006.

[40] R. Warriner, K. Johnston, and R. Cobbold, "A viscoelastic model of arterial wall motion in pulsatile flow: Implications for Doppler ultrasound clutter assessment," Physiol. Meas., vol. 29, pp. 157-179, 2008.

[41] V. Caselles, R. Kimmel, and G. Sapiro, "Geodesic active contours," Int. J. Comput. Vis., vol. 22, no. 1, pp. 61-79, 1997.

[42] R. McGill, J. Tukey, and W. Larsen, "Variations of boxplots," Amer. Statistician, vol. 32, pp. 12-16, 1978.

[43] J. Weese, T. Buzug, G. Penney, and P. Desmedt, "2D/3D registration and motion tracking for surgical interventions," Philips J. Res., vol. 51, pp. 299-316, 1998.

[44] M. Green, J. Seidel, S. Stein, T. Tedder, K. Kempner, C. Kertzman, and T. Zeffiro, "Head movement in normal subjects during simulated PET brain imaging with and without head restraint," J. Nucl. Med., vol. 35, no. 9, pp. 1538-1546, 1994.

[45] L. Love and R. Kruger, "Scatter estimation for a digital radiographic system using convolution filtering," Med. Phys., vol. 14, no. 2, pp. 178-185, 1987.

[46] M. Winslow, X. Xu, and B. Yazici, "Development of a simulator for radiographic image optimization," Comput. Meth. Programs Biomed., vol. 78, pp. 179-190, 2005.

[47] D. Xia, L. Yu, E. Sidky, Y. Zou, N. Zuo, and X. Pan, "Noise properties of chord-image reconstruction," IEEE Trans. Med. Imag., vol. 26, no. 2, pp. 1328-1344, Feb. 2007.

[48] D. Tomaževič, B. Likar, and F. Pernuš, "3D/2D image registraion: The impact of X-ray views and their number," in Proc. Int. Conf. Med. Image Comput. Computer-Assisted Intervention (MICCAI'07), 2007, pp. $450-457$. 